\title{
Percepção da Doença e Indicadores de TEPT em Mães de Sobreviventes de Câncer Infantil
}

\author{
Priscila Lawrenz \\ Universidade do Vale do Rio dos Sinos, São Leopoldo, RS, Brasil \\ Ana Carolina Wolf Baldino Peuker \\ Elisa Kern de Castro ${ }^{1}$ \\ Programa de Pós-Graduação em Psicologia da Universidade do Vale do Rio dos Sinos, \\ São Leopoldo, RS, Brasil
}

\begin{abstract}
Resumo
Objetivo: investigar a relação entre percepção da doença, transtorno de estresse pós-traumático (TEPT) e sintomatologia pós-traumática em mães de sobreviventes de câncer infantil. Método: ex post facto. Participantes: 16 mães com média de idade de 41,87 anos $(D P=8,68)$. Os questionários sobre percepção da doença (Revised Illness Perception Questionnaire for Healthy People [IPQ-RH]) e TEPT (Posttraumatic Stress Disorder Checklist - Civilian Version [PCL-C]) foram respondidos através de uma plataforma digital. Resultados: segundo os critérios de avaliação do PCL-C, oito mães (50\%) apresentaram indicadores diagnósticos de TEPT. Em relação à sintomatologia pós-traumática, 13 mães $(81,25 \%)$ apresentaram excitabilidade aumentada, 12 (75\%) revivência e oito $(50 \%)$ evitação. $\mathrm{O}$ escore global do PCL-C foi de 43 pontos $(D P=14,76)$, indicando nível moderado de sintomas de TEPT. No IPQ-RH, a dimensão representação emocional correlacionou-se positivamente com a revivência $(r=0,615 ; p<0,05)$, evitação $(r=0,679 ; p<0,01)$ e a média total do PCL-C $(r=0,631 ; p<0,01)$. A percepção de controle pessoal correlacionou-se negativamente com a evitação $(r=-0,673 ; p<0,01)$. Conclusões: a experiência de ter um filho com câncer repercute negativamente na saúde emocional das mães, tornandoas uma população vulnerável ao estresse, principalmente quando têm uma representação emocional muito negativa da doença.
\end{abstract}

Palavras-chave: Percepção da doença, Transtorno de Estresse Pós-Traumático, câncer infantil, sobreviventes, mães.

\section{IIIness Perception and Indicators of PTSD in Mothers of Childhood Cancer Survivors}

\begin{abstract}
Objective: to investigate the relationship among illness perception, posttraumatic stress disorder (PTSD) and posttraumatic symptomatology in mothers of childhood cancer survivors. Method: ex post facto design. Participants: 16 mothers with a mean age of 41.87 years old $(S D=8.68)$. The instruments used regarding the illness perception (Revised Illness Perception Questionnaire for Healthy People [IPQRH]) and PTSD (Posttraumatic Stress Disorder Checklist - Civilian Version [PCL-C]) were answered through a digital platform. Results: According to the evaluation criteria of the PCL-C, eight mothers
\end{abstract}

Endereço para correspondência: Av. Unisinos, 950, Bairro Cristo Rei, São Leopoldo, RS, Brasil 93022-000. Fone: (51) 3591-1122. E-mail: elisa.kerndecastro@gmail.com

Este artigo é parte de um projeto com apoio financeiro do Conselho Nacional de Desenvolvimento Científico e Tecnológico (CNPq). 
(50\%) obtained PTSD diagnosis indicators. Regarding the posttraumatic symptomatology, 13 mothers $(81.25 \%)$ presented increased arousal, $12(75 \%)$ reliving and eight $(50 \%)$ avoidance. Considering the global score of the PCL-C, the average was 43 points $(S D=14.76)$, indicating moderate level of PTSD indicators. Regarding the IPQ-RH, emotional representation dimension correlated positively with reliving $(r=.615 ; p<.05)$, avoidance $(r=.679 ; p<.01)$ and the total average of the PCL-C $(r=.631 ; p<.01)$. The personal control dimension correlated negatively with avoidance $(r=-.673 ; p<.01)$. Conclusions: the results showed that the experience of having a child with cancer can reflect negatively on the mental health of mothers, turning them into a population vulnerable to stress, especially when they maintain a negative emotional representation of the illness.

Keywords: Illness perception, Posttraumatic Stress Disorder, childhood câncer, survivors, mothers.

\section{Percepción de la Enfermedad y Indicadores de TEPT en Madres de Sobrevivientes de Cáncer en la Niñez}

\section{Resumen}

Objetivo: investigar la relación entre percepción de la enfermedad, Trastorno de Estrés Postraumático (TEPT) y sintomatología postraumática en madres de sobrevivientes de cáncer en la niñez. Método: ex post facto. Participantes: 16 madres con edad media de 41,87 anos $(S D=8.68)$. Los cuestionarios sobre percepción de la enfermedad (Revised Illness Perception Questionnaire for Healthy People [IPQ-RH]) y TEPT (Posttraumatic Stress Disorder Checklist - Civilian Version [PCL-C]) fueron contestados a través de una plataforma digital. Resultados: según los criterios de evaluación del PCL-C, ocho madres (50\%) presentaron indicadores diagnósticos de TEPT. En relación a la sintomatología traumática, 13 madres $(81,25 \%)$ presentaron excitación, $12(75 \%)$ reexperiencia y ocho $(50 \%)$ evitación. La puntuación global del PCL-C fue de 43 puntos ( $S D=14.76)$, lo que indica un nivel moderado de indicadores de TEPT. En lo que respecta IPQ-RH, la dimensión representación emocional se correlacionó de forma positiva con la reexperiencia $(r=.615 ; p<.05)$, evitación $(r=.679 ; p<.01)$ y la media total del PCL-C $(r=.631 ; p<.01)$. La percepción de control personal se correlacionó de forma negativa con la evitación $(r=-.673 ; p<.01)$. Conclusiones: la experiencia de tener un hijo con cáncer puede tener repercusiones negativas en la salud emocional de las madres, lo que hace con que sean una población vulnerable al estrés, principalmente cuando mantiene una representación emocional muy negativa de la enfermeda.

Palabras clave: Percepción de la enfermedad, Trastorno de Estrés Postraumático, cáncer en la niñez, sobrevivientes, madres.

Avanços importantes foram alcançados nas últimas décadas no que se refere ao desenvolvimento de novas tecnologias e tratamentos para o câncer infantil (Bruce, 2006; Silva, Teles, \& Valle, 2005). Em 1970, as chances de cura de uma criança com câncer não passavam de $30 \%$ (Andrea, 2008). Atualmente, em países desenvolvidos, cerca de $70 \%$ a $90 \%$ podem ser curados se diagnosticados e tratados precocemente (Kamibeppu et al., 2010). No Brasil, o câncer infantil representa a primeira causa de morte por doença entre crianças e adolescentes de um a 19 anos de idade. A cada ano, cerca de 11.530 novos casos surgem no país, num total de 2.740 mortes. As taxas de sobrevida ainda estão aquém do esperado, mas, em algumas regiões, podem ultrapassar 70\% (Instituto Nacional do Câncer [INCA], n.d.).

O câncer infantil corresponde a um grupo de doenças que têm como característica comum o crescimento de células anormais que afetam o funcionamento do organismo. Os tumores mais frequentes são as leucemias, os linfomas e as neoplasias do sistema nervoso central (INCA, n.d.; World Health Organization [WHO], 2014). Embora as estimativas de sobrevida sejam anima- 
doras, afirmar a cura de um câncer exige cautela, já que existe a possibilidade de recidiva da doença. O termo sobrevivente é utilizado e refere-se à parcela de pacientes que terminaram o tratamento e o câncer está controlado (Arrais \& Araújo, 2000).

Pais de sobreviventes de câncer infantil podem apresentar trauma psicológico em decorrência do alto nível de estresse associado à doença e ao envolvimento nos cuidados do filho. Alguns casos podem evoluir para um quadro de Transtorno de Estresse Pós-Traumático (TEPT; Norberg, Pöder, Ljungman, \& Essen, 2012). De acordo com isso, o conhecimento de uma doença que coloque em risco a vida de um filho foi incluído como evento traumático na quarta edição do Manual Diagnóstico e Estatístico de Transtornos Mentais (DSM-IV; American Psychiatric Association [APA], 1995).

Diferentes autores têm investigado a presença de TEPT e de sintomatologia pós-traumática nos membros da família de sobreviventes de câncer infantil. No caso das mães, estimativas apontam que $6,2 \%$ a $11 \%$ fecham diagnóstico para TEPT (Alderfer, Labay, \& Kazak, 2003) e $37 \%$ a $44 \%$ apresentam níveis elevados de sintomatologia pós-traumática (Barakat, Alderfer, \& Kazak, 2006; Bruce, Gumley, Isham, Fearon, \& Phipps, 2010). Apesar da relevância, trata-se de um tema ainda pouco estudado no Brasil, o que se confirma pela ausência de artigos nacionais abordando o assunto.

O TEPT caracteriza-se pela exposição pessoal direta a um estressor externo ou o testemunho de situações que ameacem a vida e a integridade de outra pessoa (critério A; APA, 2008). A sensação de ameaça influi no processamento cognitivo resultando em avaliações excessivamente negativas do trauma e/ou de suas consequências, além de uma elaboração pobre das memórias do evento traumático, as quais são facilmente ativadas por associações situacionais (Ehlers \& Clark, 2000). De acordo com os critérios do DSM-IV-TR (APA, 2008), os sintomas de TEPT incluem: revivência persistente do evento traumático (recordações reco rrentes e intrusivas, sonhos aflitivos nos quais o evento é reencenado; critério B); evitação de estímulos associados e entorpecimento da responsividade geral (esforços deliberados no sentido de evitar pensamentos, sentimentos, conversas, atividades e pessoas que provoquem recordações sobre o evento; critério C); excitabilidade aumentada (ansiedade, dificuldades para dormir e sobressaltos exagerados que não estavam presentes antes do trauma; critério D). O quadro completo deve estar presente por mais de um mês (critério E) e interferir no funcionamento social, ocupacional e outras áreas importantes da vida do indivíduo (critério F).

Enquanto a revivência e a excitabilidade aumentada são consideradas respostas involuntárias, a evitação configura-se como uma estratégia deliberada (Plumb, Orsillo, \& Luterek, 2004) que pode impedir a adequada elaboração do trauma (Whealin, Ruzek, \& Southwick, 2008). Neste sentido, constatou-se que pais que evitaram estímulos que geravam memórias estressantes e emoções negativas sobre o câncer dos filhos apresentaram, um ano e meio após o tratamento, mais indicadores de TEPT e níveis elevados de sintomatologia pós-traumática do que aqueles que não evitaram tais estímulos (Norberg, Pöder, \& Essen, 2011).

Partindo da ideia de que as avaliações dos pais sobre a ameaça do câncer podem impedir a elaboração do trauma, é importante investigar quais as percepções que eles têm a respeito da doença. Em Psicologia da Saúde, a percepção da doença (Ilness Perception) de pessoas doentes e saudáveis tem sido investigada seguindo o Modelo do Senso Comum (Leventhal, Brissette, \& Leventhal, 2003; Leventhal, Nerenz, \& Steele, 1984). Segundo esta teoria, estímulos de ameaça contra a saúde (p. ex., diagnóstico de câncer infantil de um filho) geram a ativação e o desenvolvimento de representações sobre determinada doença a partir de crenças individuais. As crenças são estruturadas a partir de experiências pregressas e vicárias. Tais crenças podem influenciar os comportamentos e o enfrentamento da situação (Figueiras, Machado, \& Alves, 2002). Os estímulos de ameaça ativam dois sistemas: um envolvendo processos cognitivos para regular a ameaça objetiva à saúde e outro que envol- 
ve processos emocionais para regular estresse, medo e ansiedade (Cameron \& Jago, 2008).

A princípio, a percepção da doença foi dividida em cinco dimensões: identidade (possíveis sintomas da doença), causas (fatores que dão origem à doença), duração (curso da doença ao longo do tempo, p. ex., aguda ou crônica; cíclica), consequências (gravidade da doença e seu impacto no funcionamento físico, psicológico e social) e controle/cura (possibilidade de curar ou controlar a doença; Leventhal et al., 1984). Mais tarde, a dimensão controle/cura foi dividida em controle pessoal (percepção do quanto o doente pode controlar ou curar a doença) e controle do tratamento (eficácia do tratamento no controle ou cura da doença) e duas novas dimensões foram incluídas ao modelo: coerência da doença (percepção do quanto o indivíduo compreende a doença) e representação emocional (emoções que envolvem a experiência da doença; Moss-Moris et al., 2002).

Considerando a relação entre percepção da doença e TEPT, apenas um estudo foi encontrado na literatura nacional e internacional. Trata-se de uma pesquisa realizada com pacientes cardíacos. Os resultados indicaram que a percepção da doença foi preditora de TEPT, sendo que maiores índices do transtorno associaram-se à diminuição da dimensão controle do tratamento (Sheldrik, Tarrier, Berry, \& Kincey, 2006). Em virtude da importância e do ineditismo da temática, este estudo foi delineado a fim de investigar a relação entre percepção da doença, TEPT e sintomatologia pós-traumática em mães de sobreviventes de câncer infantil. Hipotetiza-se a existência de correlações lineares positivas nas quais percepções mais negativas do câncer, avaliadas através do Revised Illness Perception Questionnaire for Healthy People (IPQ-RH), estejam associadas a maiores escores nos indicadores de TEPT e/ou sintomatologia pós-traumática (revivência, evitação e excitabilidade aumentada).

\section{Método}

\section{Delineamento}

Ex post facto (Monteiro \& Léon, 2007).

\section{Participantes}

Os critérios de participação no estudo incluíram mães de sobreviventes de câncer infantil cujos filhos receberam o diagnóstico quando tinham entre um e 19 anos de idade. A doença deveria estar em remissão e o tratamento ter sido encerrado há, pelo menos, seis meses. Das 17 mães que responderam aos instrumentos, apenas uma foi excluída porque o filho havia falecido em decorrência do câncer infantil. A média de idade das 16 participantes no momento da aplicação dos instrumentos era de 41,87 anos ( $D P=$ $8,68)$. Quando o câncer foi diagnosticado, as mães tinham média de idade de 33,80 anos $(D P=7,46)$.

\section{Instrumentos}

Questionário de Dados Sociodemográficos e Clínicos. As informações foram divididas entre dados sociodemográficos da mãe (idade atual, idade no momento do diagnóstico do filho, situação conjugal atual, escolaridade, etc.) e histórico clínico do filho sobrevivente (tempo do diagnóstico em anos, idade no momento do diagnóstico, tipo de câncer, etc.). $\mathrm{O}$ instrumento também incluiu uma questão que indagava se a mãe vivenciou, ou não, outro evento potencialmente traumático durante a sua vida (familiar com doença grave, morte de familiar, morte de alguém muito querido, acidente, violência, outro). Todos os dados foram informados pela mãe.

Posttraumatic Stress Disorder Checklist Civilian Version (PCL-C). Este instrumento é uma das três versões do PCL, desenvolvido por Weathers, Litz, Herman, Huska, e Keane (1993). O PCL-C é voltado para a população geral e não está relacionado a um evento traumático específico. Através dele, se obtém informações sobre a presença ou ausência de indicadores de TEPT e sintomatologia pós-traumática. Trata-se de um instrumento de autorrelato formado por 17 itens baseados nos critérios estabelecidos pelo DSM-IV (APA, 1995) para diagnóstico de Transtorno de Estresse Pós-Traumático (TEPT). Os respondentes devem indicar o quanto têm sido perturbados no último mês pelos sintomas descritos no instrumento utilizando uma escala Likert de um (nada) a cinco (muito). A avaliação do ins- 
trumento pode ser realizada por agrupamento (clusters) ou escore global (ponto de corte). No agrupamento, o participante apresenta TEPT se pontuar três ou mais em, pelo menos, um item de revivência (critério B - questões 1 a 5), três itens de evitação (critério $\mathrm{C}$ - questões 6 a 12) e dois itens de excitabilidade aumentada (critério D - questões 13-17; International Society for Traumatic Stress Studies [ISTSS], 2014). A segunda forma de avaliação é através do ponto de corte maior ou igual a 40 (Schwartz et al., 2012). O PCL-C foi utilizado em um estudo com sobreviventes de câncer infantil (Schwartz \& Drotar, 2006) e a versão original em inglês apresenta consistência interna de 0,96. No Brasil, o instrumento foi validado em indivíduos que vivenciaram acidentes de trânsito (Bringhenti, Luft, \& Oliveira, 2010). Sua equivalência semântica para o português foi constatada por Berger, Mendlowicz, Souza, e Figueira (2004) e utilizada na presente pesquisa.

Revised Illness Perception Questionnaire for Healthy People (IPQ-RH). Instrumento desenvolvido por Figueiras e Alves (2007) a partir do Revised Illness Perception Questionnaire (IPQ-R; Weinman, Petrie, Moss-Moris, \& Horne, 1996) para avaliar a percepção da doença em indivíduos saudáveis de acordo com o Modelo do Senso Comum. O instrumento possui adaptação para o português e está dividido em três sessões: a primeira corresponde à dimensão identidade, onde são apresentados sintomas (p. ex., dores, falta de ar, fadiga, etc.). As participantes são convidadas a responder se acreditam, ou não, que esses sintomas estão relacionados à doença (neste caso, ao câncer infantil; Sim = 1; Não = 2). A soma do número de respostas "Sim" determina o escore global da subescala identidade. A segunda sessão envolve as dimensões duração aguda/crônica, consequências, controle pessoal, controle do tratamento, coerência, duração cíclica e representação emocional. A terceira sessão relaciona-se às possíveis causas da enfermidade, divididas em atribuições psicológicas e gerais. É solicitado, também, que a participante enumere, livremente, três causas que considere estar relacionadas com o desenvolvimento da doença.
A segunda e terceira sessões contemplam uma escala Likert que vai da opção concordo plenamente até discordo plenamente.

\section{Procedimentos de Coleta de Dados}

A coleta de dados foi realizada pela internet através de um formulário autoaplicável elaborado na ferramenta Google Docs. Um site foi criado para divulgar a pesquisa, apontar os critérios de inclusão e informar sobre a aplicação dos instrumentos (link do site da pesquisa: http://pesquisa-unisinos-ici-familiares.webnode. com/). Um link disponibilizado no site gerava uma nova página com o Termo de Consentimento Livre e Esclarecido (TCLE). As participantes só respondiam aos instrumentos depois de confirmarem a leitura e a aceitação do TCLE. Caso não aceitassem participar, uma nova mensagem com um agradecimento era apresentada e a coleta era encerrada. Uma cópia do TCLE foi enviada por e-mail para cada uma das respondentes, as quais disponibilizaram um endereço eletrônico na mesma página em que foi apresentado o TCLE. As coletas online ocorreram no período de agosto de 2013 a março de 2014.

A divulgação do site da pesquisa ocorreu através das redes sociais (Facebook), nos meios de divulgação eletrônica (site) do Instituto do Câncer Infantil (ICI-RS) e a partir de cartazes distribuídos no instituto. Além disso, foi realizada uma busca ativa através de listas disponibilizadas pelo ICI-RS, o qual mantém cadastros de sobreviventes e seus familiares. Ligações telefônicas foram realizadas por pesquisadoras treinadas, graduandas em Psicologia, com o intuito de convidar as mães de sobreviventes. Às interessadas em participar do estudo, as pesquisadoras disponibilizavam o link do site da pesquisa, que era enviado por e-mail.

O método de coleta de dados, através da internet, foi determinado em virtude da dificuldade de contato presencial com as mães dos sobreviventes no ICI-RS, localizado em Porto Alegre. Para viabilizar o tratamento do filho na capital, muitas famílias deixam suas cidades no interior do estado temporariamente. Com o término do acompanhamento médico e a volta para casa, o 
contato sistemático com o ICI-RS diminui. Este aspecto tornou o acesso às famílias dificultado $\mathrm{e}$ os contatos passaram a ser realizados pelo telefone e redes sociais.

\section{Procedimentos Éticos}

Projeto aprovado pelo Comitê de Ética da Universidade do Vale do Rio dos Sinos sob o número 13962113.8.1001.5344. Foram adotados os procedimentos éticos para pesquisa com seres humanos. As páginas da web contendo o TCLE e os instrumentos, bem como o banco de dados com as informações coletadas, foram armazenados e protegidos por senha com o objetivo de garantir a privacidade e a segurança das informações. Todas as participantes aceitaram responder à pesquisa e receberam uma cópia do TCLE enviada para o seu $e$-mail.

\section{Análise dos Dados}

Inicialmente, foram realizadas análises descritivas para verificar médias, desvios-padrão, frequências e percentagens. Em seguida, análises de correlações de Spearman foram efetuadas para investigar a possível relação entre as dimensões do IPQ-RH e os sintomas do PCL-C. Em todas as análises foi adotado nível de significância de 5\% e conduzidas utilizando o pacote estatístico SPSS (Statistical Package for the Social Sciences) versão 20.0.

Também foram realizadas análises de conteúdo (Bardin, 1977) para a codificação das respostas obtidas da questão aberta "Por favor, classifique por ordem de importância os três fatores que lhe parecem poder causar o câncer infantil" contida no instrumento do IPQ-RH. Inicialmente, as respostas foram descritas na íntegra e, posteriormente, agrupadas a partir do conteúdo.

\section{Resultados}

Em relação aos dados sociodemográficos das participantes, a maioria declarou ser casada ou viver com o companheiro $(56,25 \% ; n=9)$. Quanto à escolaridade e atividade laboral, 10 $(62,5 \%)$ estudaram até ensino médio completo e trabalhavam. Metade das mães contou com acompanhamento psicológico durante o tratamento de câncer do filho e apenas duas $(12,5 \%)$ estavam em acompanhamento com psicólogo quando responderam à pesquisa. A maioria das mães $(87,5 \%)$ afirmou ter vivenciado outro evento potencialmente traumático durante a vida. Destes, "morte" e "doença grave de um familiar" foram os mais prevalentes.

Quanto ao histórico clínico do filho sobrevivente de câncer infantil, o tipo de câncer mais frequente foi a Leucemia $(56,25 \% ; n=9)$. Em relação ao tratamento, todos realizaram quimioterapia, seis radioterapia, sete cirurgia e dois transplante de medula. De acordo com as mães, quatro sobreviventes desenvolveram sequelas físicas decorrentes da doença ou do tratamento. Quatro sobreviventes tiveram recidiva, mas nenhum desenvolveu outro tipo de câncer mais tarde. A média de idade dos filhos no momento do diagnóstico da doença era de 6,08 anos $(D P=$ $3,98)$, o tratamento teve duração de 28,33 meses $(D P=20,55)$ e o diagnóstico havia sido realizado há 7,80 anos $(D P=5,71)$.

De acordo com o critério de agrupamento do PCL-C, oito (50\%) mães apresentaram indicadores de diagnóstico de TEPT. Em termos de sintomatologia, $13(81,25 \%)$ apresentaram excitabilidade aumentada, $12(75 \%)$ reportaram revivência e oito $(50 \%)$ evitação. Considerando o escore global do PCL-C, a média foi de 43 pontos $(D P=14,76)$, indicando nível moderado de TEPT. A avaliação, segundo o ponto de corte ( $\geq 40$ pontos), indicou que $10(62,5 \%)$ mães apresentaram sintomas que caracterizavam a presença de TEPT. As médias dos escores permitiram identificar níveis moderados dos sintomas excitabilidade aumentada $(M=15,31 ; D P=6,49)$, evitação $(M=15,31 ; D P=5,66)$ e revivência $(M$ $=12,38 ; D P=4,92)$.

Os resultados da primeira sessão da percepção da doença, relacionada à dimensão identidade, demonstraram que as mães percebiam o câncer infantil como uma doença muito sintomática, sendo que os sintomas mais frequentemente relacionados foram: dores $(93,75 \%)$, perda de peso $(81,25 \%)$, fadiga $(81,25 \%)$, perda de forças $(81,25 \%)$, dor de cabeça $(75 \%)$, indisposição do estômago (75\%), tonturas $(68,75 \%)$ e náusea 
$(62,5 \%)$. As dimensões da segunda sessão foram interpretadas considerando um intervalo de variação de um a cinco de acordo com a escala Likert (Discordo Plenamente a Concordo Plenamente). A média da duração aguda/crônica, acima de três, indicou que as mães percebiam o câncer infantil como uma doença mais crônica do que aguda. Quanto às consequências, a média foi acima de quatro, ou seja, a doença era percebida como tendo graves consequências. Em relação ao controle pessoal e controle do tratamento, as médias apontaram que as mães consideraram que tanto os comportamentos do doente quanto o tratamento podem ser eficazes no controle do câncer infantil. A média 3,06 da dimensão coerência indicou que as mães consideravam que compreendiam parcialmente a doença. O câncer infantil também foi considerado como uma doença cíclica $(M=3,85 ; D P=0,54)$. A média da representação emocional, próxima de quatro, apontou que as mães consideravam a doença como grave e ameaçadora.

Tabela 1

Média, Desvio-Padrão, Mínimo e Máximo das Pontuações Relativas à Sintomatologia de TEPT (PCL-C) e Percepção da Doença (IPQ-RH)

\begin{tabular}{|c|c|c|c|c|c|}
\hline & Média & $D P$ & Mínimo & Máximo & Variação \\
\hline Revivência & 12,3 & 4,9 & 05 & 21 & $05-25$ \\
\hline Evitação & 15,3 & 5,6 & 08 & 25 & $07-35$ \\
\hline Excitabilidade & 15,3 & 6,4 & 05 & 25 & $05-25$ \\
\hline Total PCL-C & 43,0 & 14,7 & 22 & 71 & $17-85$ \\
\hline Duração aguda/crônica & 3,3 & 0,5 & 2,6 & 4,6 & $01-05$ \\
\hline Consequências & 4,2 & 0,5 & 3,2 & 5,0 & $01-05$ \\
\hline Controle pessoal & 3,5 & 0,9 & 3,5 & 5,0 & $01-05$ \\
\hline Controle do tratamento & 4,1 & 0,4 & 3,3 & 5,0 & $01-05$ \\
\hline Coerência & 3,0 & 0,9 & 1,6 & 4,3 & $01-05$ \\
\hline Duração cíclica & 3,8 & 0,5 & 2,6 & 4,6 & $01-05$ \\
\hline Representação emocional & 3,9 & 0,8 & 2,0 & 5,0 & $01-05$ \\
\hline
\end{tabular}

$\mathrm{Na}$ terceira sessão do IPQ-RH, referente às possíveis causas do câncer infantil, as médias indicaram que as mães atribuíram mais causas gerais (p. ex., hereditariedade, tipo de alimentação, destino ou má sorte; $M=23,44 ; D P=2,82$ ) do que psicológicas (p. ex., estresse ou preocupação, estado emocional, personalidade; $M=$ $10,12 ; D P=2,52)$ ao surgimento da doença. As respostas da pergunta aberta "Por favor, classifique por ordem de importância os três fatores que lhe parecem poder causar o câncer infantil" foram analisadas e categorizadas. As categorias foram adaptadas a partir do estudo de Figueiras e Alves (2007):

1. Dieta/Hábitos alimentares/Excesso de peso;
2. Dificuldade ao acesso em relação ao cuidado médico;

3. Questões biológicas/Hormônios/Imunidade;

4. Hereditariedade/Genética;

5. Atitude pessoal/Comportamento;

6. Estado emocional/Estresse/Preocupações;

7. Excesso de trabalho;

8. 8. Crenças místicas ou religiosas;

9. Não sabe;

10. Outras. Na Tabela 2 são apresentadas as principais causas referidas pelas mães, sendo que Hereditariedade/Genética e Questões biológicas/Hormônios/Imunidades foram as mais prevalentes. 
Tabela 2

Principais Causas Atribuídas pelas Mães para o Desenvolvimento do Câncer Infantil no Filho

\begin{tabular}{lccc}
\hline & \multicolumn{2}{c}{ Ordem de atribuição } \\
\cline { 2 - 4 } Causas & Primeira (\%) & Segunda (\%) & Terceira (\%) \\
\hline Hereditariedade/Genética & 43,75 & 25 & 12,5 \\
Questões biológicas/Hormonais/Imunidade & 25 & 18,75 & 31,25 \\
Dieta/Hábitos alimentares/Excesso de peso & 12,5 & 6,25 & - \\
Crenças místicas ou religiosas & - & 12,5 & 12,5 \\
Estado emocional/Estresse/Preocupações & - & 6,25 & 6,25 \\
Dificuldade ao acesso em relação ao cuidado médico & - & - & 6,25 \\
Outras & 18,75 & 12,5 & 31,25 \\
Não sabe & - & 18,75 &
\end{tabular}

A análise de correlação de Spearman, realizada para examinar a relação entre as dimensões da percepção da doença (duração aguda/ crônica, consequências, controle pessoal, controle do tratamento, coerência, duração cíclica e representação emocional) e os sintomas de TEPT (revivência, evitação e excitabilidade aumentada), indicou que, quanto mais negativa a representação emocional do câncer infantil, mais sintomas de revivência $(0,615 ; p<0,05)$ e evitação $(0,679 ; p<0,01)$. Quanto menor a percepção de controle pessoal, mais sintomas de evitação $(-0,673 ; p<0,01)$. Da mesma forma, quanto mais negativa a representação emocional do câncer infantil, maior o escore total do PCL-C $(0,631$; $p<0,01)$. A subescala excitabilidade aumentada não se correlacionou com as dimensões da percepção da doença.

\section{Discussão}

Os resultados permitiram identificar relações significativas entre percepção da doença, TEPT e sintomatologia pós-traumática. A dimensão representação emocional correlacionou-se positivamente com os sintomas evitação, revivência e a média total do PCL-C. Quanto mais negativas as percepções sobre as emoções associadas à doença, mais sintomas de TEPT eram apresentados, maiores as tentativas de evitar estímulos relacionados ao evento e maior a presença de lembranças intrusivas sobre a expe- riência. A dimensão controle pessoal se correlacionou negativamente com o sintoma evitação, sugerindo que, quanto menor a percepção de controle e cura do câncer, maiores eram as tentativas de evitar pensamentos e atividades que provocassem recordações sobre o evento.

O Modelo do Senso Comum propõe que a regulação emocional desempenha um papel importante na gestão do estresse (Cameron \& Jago, 2008). Os resultados apontaram que as mães percebiam o câncer infantil como uma ameaça que gerava muito medo e ansiedade. A evitação parece ter sido empregada como uma estratégia para lidar com os estados emocionais negativos associados à experiência do câncer do filho. A evitação pode ser considerada uma estratégia de enfrentamento efetiva em curto prazo, pois proporciona alívio temporário. Porém, em longo prazo, as tentativas de afastar emoções negativas acabam tornando-as mais intensas e dificultando a elaboração das memórias traumáticas. A evitação também pode repercutir negativamente nos cuidados da saúde do filho com câncer. Há evidências de que pais de sobreviventes de câncer infantil podem evitar consultas médicas de acompanhamento como um esforço de afastar o estresse negativo que a situação acarreta (Norberg et al., 2011).

Os achados reforçam a noção de que mães de sobreviventes de câncer infantil constituem um grupo altamente vulnerável ao estresse (Norberg et al., 2012). Indicadores de níveis mode- 
rados de TEPT foram identificados em $50 \%$ e $62,5 \%$ da amostra de acordo com os critérios de avaliação utilizados, sendo que os índices encontrados na literatura internacional variam de $6,2 \%$ a $11 \%$ para presença de TEPT (Alderfer et al., 2003) e $37 \%$ a $44 \%$ para manifestação de sintomas (Barakat et al., 2006; Bruce et al., 2010). Lee e Santacroce (2007) apontam para a importância de investigar não apenas a possível presença do transtorno, mas, também, para a existência de sintomatologia. Apresentar um dos grupos de sintomas já representa sofrimento, dificuldades e prejuízos em diferentes âmbitos da vida do indivíduo. Em termos de prevalência, $75 \%$ das mães apresentaram revivência, indicando que, mesmo após meses ou anos do término do tratamento, elas podem ter recordações recorrentes e mesmo sonhos aflitivos que envolvem a experiência traumática. Metade delas referiu sintomas de evitação, caracterizados pelo afastamento de pensamentos e atividades relacionadas ao evento traumático. Os sintomas de excitabilidade aumentada, encontrados em 81,25\% da amostra, indicaram a presença de ansiedade e hipervigilância.

As distinções entre as prevalências observadas neste estudo e os índices encontrados em pesquisas internacionais podem estar relacionadas a aspectos socioculturais, instrumentos utilizados, parâmetros de avaliação e tamanho amostral. Além do número reduzido de participantes, a amostra caracterizou-se pela heterogeneidade (mães com filhos acometidos por diferentes tumores, submetidos a tratamentos diversos, com vivências de outros eventos traumáticos, etc.). Em decorrência disso, os resultados devem ser interpretados de forma cautelosa, assim como a extrapolação dos achados para outras populações. Novos estudos devem considerar esses aspectos no sentido de aprimorar a análise das correlações, podendo encontrar outras evidências relevantes e úteis para a compreensão aprofundada da população investigada.

Embora o PCL-C tenha sido útil na identificação de possíveis casos de TEPT, para a confirmação do diagnóstico é imprescindível a avaliação clínica por profissional capacitado. A maioria das mães $(87,5 \%)$ afirmou ter vivenciado outro evento potencialmente traumático du- rante a vida. Destes, "morte" e "doença grave de um familiar" foram os mais prevalentes. Embora as participantes tenham sido orientadas a responder aos instrumentos relacionando suas respostas ao câncer infantil, outros eventos estressores podem ter influenciado os resultados. Existem variáveis predisponentes, como traços de personalidade ou antecedentes causais que diminuem o limiar para a manifestação da sintomatologia ou agravam sua evolução. Por isso, além do emprego de escalas ou questionários, é importante a realização de uma investigação clínica para que a presença do transtorno seja bem estabelecida (Bringhenti et al., 2010).

Embora tenha sido alta, a prevalência e a intensidade da sintomatologia de TEPT ainda podem ter sido subestimadas. É possível que mães com sintomas mais graves tenham evitado responder aos instrumentos da pesquisa no intuito de esquivarem-se de conteúdos percebidos como negativos, ameaçadores e carregados emocionalmente. Este aspecto também pode ter repercutido no tamanho amostral reduzido.

Em relação à percepção da doença, avaliada através do IPQ-RH, constatou-se que as mães percebiam o câncer infantil como muito sintomático. É possível que os efeitos adversos causados pelo tratamento do filho podem ter sido considerados, já que todos realizaram quimioterapia e um número significativo realizou radioterapia e cirurgia. A quimioterapia, por exemplo, pode causar efeitos colaterais como náuseas, vômitos e fadiga.

Quanto às consequências, as mães consideraram que o câncer impõe muitas dificuldades ao doente e às pessoas próximas dele. Esta percepção se coaduna ao contexto no qual se insere a experiência do câncer infantil. Ao sofrimento imposto pela descoberta da doença e pelos efeitos do tratamento, somam-se problemas de ordem doméstica, como gerenciar o funcionando da casa, cuidar dos outros filhos e do casamento. De forma comum, as mães precisam se ausentar do trabalho ou afastam-se por não ter disposição para realizar suas tarefas em virtude das preocupações com o filho doente. Além disso, a família tende a enfrentar dificuldades financeiras, já que os tratamentos são caros e realizados em grandes centros urbanos. Pacientes e familiares residen- 
tes de outras cidades precisam deixar suas casas para permanecer por longos períodos no hospital (Andrea, 2008; Ortiz, 2003).

As mães também consideraram o câncer infantil uma doença crônica e cíclica. A noção de cronicidade do câncer infantil envolve a percepção de que ele dura muito e, mesmo após o tratamento bem-sucedido, há possibilidade de que a doença reapareça e não tenha cura. Outra ideia é de que os sintomas vêm e vão em ciclos, tornando o câncer infantil imprevisível. No que refere às dimensões controle pessoal e controle do tratamento, as mães acreditavam que as atitudes e comportamentos do filho doente poderiam determinar o curso da doença. $\mathrm{O}$ tratamento foi percebido como eficaz no controle/cura do câncer infantil. Essas percepções parecem coerentes com o fato de que seus filhos enfrentaram o câncer e o tratamento contribuiu para torná-los sobreviventes. De forma oposta, é possível que mães que perderam seus filhos por causa do câncer tenham percepções de controle pessoal e do tratamento diminuídas.

A análise da dimensão coerência demonstrou que as mães consideraram que não compreendiam totalmente o câncer infantil. A literatura específica da área indica que uma das perguntas mais frequentes que os pais fazem é: Por que o meu filho tem câncer? As explicações da equipe médica sobre a doença e o tratamento, embora importantes, não são, em geral, suficientes para aplacar as ansiedades da família (Ortiz, 2003).

Quanto à representação emocional, as mães consideraram o câncer infantil uma doença que gerava medo, ansiedade e ameaça à vida. Apesar do aumento da sobrevida de crianças e adolescentes com câncer, a doença ainda carrega o estigma social de que leva, invariavelmente, à morte (Ortiz, 2003). Ao descobrir o câncer do filho, a mãe pode experimentar raiva, dor ou negação. Posteriormente, tendem a aparecer sintomas depressivos e a culpa por não conseguir protegê-lo do sofrimento (Méndez, Orgilés, López-Roig, \& Espada, 2004). Com o término do tratamento, as mães podem apresentar um medo exagerado de que o filho adoeça novamente (Perina, Mastellaro, \& Nucci, 2008), o que corrobora os achados deste estudo.
Quanto às causas do câncer, as mães atribuíram mais causas gerais (p. ex., hereditariedade, tipo de alimentação, destino ou má sorte) do que psicológicas (p. ex., estresse ou preocupação, estado emocional, personalidade). Quando o câncer acomete um adulto, a mídia, o senso comum e os próprios profissionais da saúde costumam atribuir o surgimento a fatores como o estilo de vida do paciente (p. ex., tabagismo, má alimentação, sedentarismo, etc.) e ao estado emocional (p. ex., excesso de preocupações). No caso do câncer infantil, não há um consenso científico sobre as causas, dificultando a prevenção (INCA, n.d.) e a compreensão por parte da família (Andrea, 2008).

\section{Conclusão}

Este trabalho propiciou, de forma pioneira no contexto nacional, maior entendimento sobre a relação entre percepção da doença, TEPT e sintomatologia pós-traumática em mães de sobreviventes de câncer infantil. Os resultados permitiram confirmar a hipótese de que percepções muito negativas sobre o câncer infantil podem estar associadas aos sintomas evitação, revivência e à média total do PCL-C. A alta prevalência de sintomatologia pós-traumática e indicadores de diagnóstico de TEPT evidenciaram o sofrimento psicológico e a alta vulnerabilidade desse grupo. A relevância clínica dos resultados reforça a necessidade de desenvolvimento de intervenções e estratégias de cuidado direcionadas a mães de sobreviventes de câncer infantil. Nesse sentido, a atuação da Psicologia não deve se restringir ao momento do diagnóstico e ao período do tratamento, visto que repercussões negativas da experiência podem acompanhar as mães mesmo após o término de um tratamento exitoso.

Os resultados desta investigação devem ser interpretados com cautela em função do número reduzido de participantes e da não aleatoriedade da amostra. Por se tratar de um estudo transversal, não se determinou causalidade. O ineditismo do tema no Brasil sugere que novos estudos, com delineamentos mais robustos, sejam desenvolvidos para investigar a presença de TEPT e sua relação com outras variáveis relevantes em 
mãe de sobreviventes. Por exemplo, a associação com condições comórbidas predisponentes, como depressão e ansiedade. Em novos estudos, as mudanças propostas pelo DSM-5 para TEPT devem ser consideradas. Além disso, protocolos de intervenção direcionados à prevenção de TEPT em mães com filhos diagnosticados com câncer podem ser desenvolvidos e testados em novas pesquisas.

\section{Referências}

Alderfer, M. A., Labay, L. E., \& Kazak, A. E. (2003). Brief report: Does posttraumatic stress apply to siblings of childhood cancer survivors? Journal of Pediatric Psychology, 28(4), 281-286. doi:10.1093/jpepsy/jsg016

American Psychiatric Association. (1995). DSM-IV ${ }^{T M}$ : Manual diagnóstico e estatístico de transtornos mentais (4. ed., M. R. Jorge, Consultoria \& Coordenação). Porto Alegre, RS: Artes Médicas.

American Psychiatric Association. (2008). DSM$-I V-T R^{T M}$ : Manual diagnóstico e estatístico de transtornos mentais: Texto revisado (4. ed., M. R. Jorge, Consultoria \& Coordenação). Porto Alegre, RS: Artes Médicas.

Andrea, M. L. M. de. (2008). Oncologia pediátrica. In V. A. de Carvalho (Ed.), Temas em psico-oncologia (pp. 477-495). São Paulo, SP: Sumus.

Arrais, A. R., \& Araújo, T. C. (2000). Recidiva X cura: A vivência paradoxal da sobrevivência ao câncer na infância. Revista Brasileira de Cancerologia, 45(3), 9-19, 2000. Recuperado em http:// www.inca.gov.br/rbc/n 45/v03/artigo2.html

Barakat, L. P., Alderfer, M. A., \& Kazak, A. E. (2006). Posttraumatic growth in adolescent survivors of cancer and their mothers and fathers. Journal of Pediatric Psychology, 32(4), 413419. doi:10.1093/jpepsy/jsg016

Bardin, L. (1977). Análise de conteúdo. Lisboa, Portugal: Edições 70.

Berger, W., Mendlowicz, M. V., Souza, W. F., \& Figueira, I. (2004). Equivalência semântica da versão em português da Post-traumatic Stress Disorder Checklist - Civilian Version (PCL-C) para rastreamento do transtorno de estresse pós-traumático. Revista de Psiquiatria do Rio Grande do Sul, 26(2), 167-175. doi:10.1590/ S0101-81082004000200006

Bringhenti, M. E., Luft, C. D. B., \& Oliveira, W. F. (2010). Transtorno de estresse pós-traumático em acidentes de trânsito: Validação da escala. Psico-USF, 14(2), 193-203. doi:10.1590/S141382712010000200007

Bruce, M. (2006). A systematic and conceptual review of posttraumatic stress in childhood cancer survivors and their parents. Clinical Psychology Review, 26, 233-256. doi:10.1016/j.cpr.2005.10.002

Bruce, M., Gumley, D., Isham, L., Fearon, P., \& Phipps, K. (2010). Post-traumatic stress symptoms in childhood brain tumour survivors and their parents. Child: Care, Health and Development, 37(2), 244-251. doi:10.1111/j.13652214.2010.01164.x

Cameron, L. D., \& Jago, L. (2008). Emotion regulation interventions: A common-sense model approach. British Journal of Health Psychology, 13, 215221. doi:10.1348/135910708X288800

Ehlers, A., \& Clark, D. M. (2000). A cognitive model of posttraumatic stress disorder. Behavior Research and Therapy, 38(4), 316-145. doi:10.1016/S0005-7967(99)00123-0

Figueiras, M. J., \& Alves, N. C. (2007). Lay perceptions of serious illnesses: An adapted version of the Revised Illness Perception Questionnaire (IPQ-R) for healthy people. Psychology and Health, 22(2), 143-158. doi:10.1080/14768320600774462

Figueiras, M. J., Machado, V. A., \& Alves, N. C. (2002). Os modelos do senso-comum das cefaleias crónicas nos casais: Relação com o ajustamento marital. Análise Psicológica, 20(1), 77-90. Recuperado em http://www. scielo.oces.mctes.pt/scielo.php?pid=S0870$-82312002000100008 \&$ script $=$ sci_arttext

Instituto Nacional do Câncer. (n.d.). Câncer - $O$ $q u e$ é. Rio de Janeiro, RJ: Autor. Recuperado em http://www2.inca.gov.br/wps/wcm/connect/ cancer/site/oquee

International Society for Traumatic Stress Studies. (2014). Assessing trauma - Posttraumatic Stress Disorder Checklist (PCL). Deerfield, IL: Author. Retrieved from http://www.istss.org//AM/ Template.cfm?Section=Home

Kamibeppu, K., Sato, I., Honda, M., Ozono, S., Sakamoto, N., Iwai, T, ...Ishida, Y. (2010). Mental health among young adult survivors of childhood cancer and their siblings including posttraumatic growth. Journal of Cancer Survivorship, 4, 303312. doi:10.1007/s11764-010-0124-z.

Lee, Y., \& Santacroce, S. J. (2007). Posttraumatic stress in long-term young adult survivors of childhood cancer: A questionnaire survey. In- 
ternational Journal of Nursing Studies, 44(8), 1406-1477. doi:10.1016/j.ijnurstu.2006.07.002

Leventhal, H., Brissette, I., \& Leventhal, E. A. (2003). The common-sense model of self-regulation of health and illness. In L. D. Cameron \& H. Leventhal (Eds.), The self-regulation of health and illness behavior (pp. 42-61). London: Routledge.

Leventhal, H., Nerenz, D., \& Steele, D. J. (1984). Illness representation and coping with health threats. In A. Baum, T. A. Revenson, \& J. E. Singer (Eds.), Handbook of Health Psychology (pp. 219-252). London: Lawrence Erlbaum.

Méndez, X., Orgiléz, M., López-Roig, S., \& Espada, J. P. (2004). Atención psicológica en el cáncel infantil. Psicooncología, 1(1), 139-154. Recuperado en http://revistas.ucm.es/index.php/PSIC/ article/view/PSIC0404110139A

Monteiro, I., \& Léon, O. G. (2007). A guide for naming research studies in Psychology. International Journal of Clinical and Health Psychology, 7(3), 847-862. Retrieved from http://www.redalyc. org/articulo.oa? $\mathrm{id}=33770318$

Moss-Moris, R., Weinman, J., Petrie, K., Horne, R., Cameron, L., \& Buick, D. (2002). The revised illness perception questionnaire (IPQR). Psychology and Health, 17(1), 1-16. doi:10.1080/08870440290001494

Norberg, A. L., Pöder, U., \& Essen, L. V. (2011). Early avoidance of disease and treatment related distress predicts post-traumatic stress in parents of children with cancer. European Journal of Oncology Nursing, 15(1), 80-84. doi:10.1016/j.ejon.2010.05.009

Norberg, A. L., Pöder, U., Ljungman, G., \& Essen, L. (2012). Objective and subjective factors of predictors of post-traumatic stress symptoms in parents of children with cancer - A longitudinal study. PlosOne, 7(5), 1-8. doi:10.1371/journal. pone. 0036218

Ortiz, M. C. M. (2003). À margem do leito: A mãe e o câncer infantil. São Paulo, SP: Arte e Ciência.

Perina, E. M., Mastellaro, M. J., \& Nucci, N. A. G. (2008). Efeitos tardios do tratamento do câncer na infância e na adolescência. In V. A. Carvalho (Ed.), Temas em psico-oncologia (pp. 496-504). São Paulo, SP: Sumus.

Plumb, J. C., Orsillo, S. M., \& Luterek, J. A. (2004). A preliminary test of the role of experiential avoidance in post-event functioning. Journal of Behavior Therapy and Experimental Psychiatry, 35(3), 245-257. doi:10.1016/j.jbtep.2004.04.011
Schwartz, L., \& Drotar, D. (2006). Posttraumatic stress and related impairment in survivors of childhood cancer in early adulthood compared to healthy peers. Journal of Pediatric Psychology, 31(4). doi:10.1093/jpepsy/jsj018

Schwartz, L. A., Kazak, A. E., Derosa, B. W., Hocking, M. C., Hobbie, W. L., \& Ginsberg, J. P. (2012). The role of beliefs in the relationship between health problems and posttraumatic stress in adolescent and young adult cancer survivors. Journal of Clinical Psychology in Medical Settings, 19(2), 138-146. doi:10.1007/s10880-011-9264-1

Sheldrik, R., Tarrier, N., Berry, E., \& Kincey, J. (2006). Post-traumatic stress disorder and illness perceptions over time following myocardial infarction and subarachnoid haemorrhage. British Journal of Health Psychology, 11(3), 387-400. doi:10.1348/135910705X71434

Silva, G. M. da, Teles, S. S., \& Valle, E. R. M. do. (2005). Estudo sobre as publicações brasileiras relacionadas a aspectos psicossociais do câncer infantil - Período de 1998 a 2004. Revista Brasileira de Cancerologia, 51(3), 253-261. Recuperado em http://www.inca.gov.br/rbc/n_51/v03/ pdf/revisao4.pdf

Weathers, F. W., Litz, B. T., Herman, D. S., Huska, J. A., \& Keane, T. M. (1993). The PTSD Checklist (PCL): Reliability, validity, and diagnostic utility. Annual Meeting of International Society for Traumatic Stress Studies. San Antonio, TX: International Society for Traumatic Stress Studies. Retrieved from http://www.istss.org/ PosttraumaticStressDisorderChecklist.htm

Weinman, J., Petrie, K. J., Moss-Moris, R., \& Horne, R. (1996). The illness perception questionnaire: A new method for assessing the cognitive representation of illness. Psychology and Health, 11(3), 431-445. doi:10.1080/08870449608400270

Whealin, J. M., Ruzek, J. I., \& Southwick, S. (2008). Cognitive-behavioral theory and preparation for professionals at risk for trauma exposure. Trauma, Violence \& Abuse, 9(2), 100-113. doi:10.1177/1524838008315869.

World Health Organization. (2014). Cancer. Geneva, Switzerland: Author. Retrieved from http:// www.who.int/topics/cancer/en/

Recebido: 10/10/2014

$1^{a}$ revisão: 18/02/2015

$2^{a}$ revisão: $22 / 04 / 2015$

Aceite final: $24 / 04 / 2015$ 